\title{
Beyond Lisbon: \\ Demographic trends and voting power in the European Union Council of Ministers
}

\author{
László Á. Kóczy* \\ Institute of Economics, Hungarian Academy of Sciences, Budaörsi út 45., H-1112 \\ Budapest, Hungary and Keleti Faculty of Business and Management, Óbuda University, \\ Tavaszmezõ 15-17. H-1084 Budapest
}

\begin{abstract}
Decision making has become slow in the 27-member European Union and the Treaty of Nice distributed power in a somewhat arbitrary way. The Lisbon Treaty makes decision making easier, and streamlines the process by removing the most controversial element: the voting weights. The new system relies entirely on population data. We look at the immediate impact of the reform as well as the long term effects of the different demographic trends across countries. We find that the Lisbon rules hurt medium sized countries, especially Central Eastern European countries with declining populations most, while the United Kingdom is the clear winner.
\end{abstract}

Keywords: European Union Council of Ministers, qualified majority voting, power index, a priori voting power, demographics

*The paper has benefited from comments from Gábor Tóka and seminar participants at the Institute of Economics, Hungarian Academy of Sciences. The author thanks the funding of the OTKA (Hungarian Fund for Scientific Research, NF-72610), the European Commission (PERG-GA-2008-230879), and the Hungarian Academy of Sciences under its Momentum Programme (LD-004/2010).

Email address: koczy@iehas.hu (László Á. Kóczy)

The final publication is available at Elsevier via http://dx.doi.org/10.1016/j.mathsocsci.2011.08.005 


\section{Introduction}

The European Union, with 27 members it is one of the largest and economically most powerful unions on the globe. ${ }^{1}$ Unfortunately the efforts to deepen the cooperation have been hampered by the complicated decision making processes paralysed by safety measures to ensure that no group of countries can be exploited by others. The Council of Ministers (CM) is the main decision making body uses qualified majority voting with conditions on the total (weighted) votes, the total population and the number of supporting countries is met.

While the entire voting process has been subject to criticism, the first condition is especially controversial. The weighted votes have been assigned to express the differences in country sizes, but the actual weights are rather arbitrary. While in 1958 Belgium and the Netherlands each had approximately 10 million inhabitants, and were thus treated equally, this remained so until recently despite a difference of over $60 \%$ in current populations. Such changes require the continuous updating of the voting weights keeping member countries in a persistent debate.

The Treaty of Lisbon attempts to solve the two issues at once. The idea was to remove the voting weights and create a voting system that is both fair, simple and requires no adjustments should the EU accept new members or should the populations change dramatically.

In this note we look at the effect of the voting reform on the individual countries' influence on decision making both on the short and long term. Our long term calculations are based on the population forecasts of Eurostat. Ours is not the first to analyse the new voting system in the Council, but unlike Felsenthal and Machover (2007), Turnovec (2008) or Barr and Passarelli (2009) we look also at the long term effects using population estimates. Since the voting rules under the Lisbon treaty are based on population data, voting power is very sensitive to these values.

The Lisbon treaty changes the decision making in other aspects as well. It gives a greater influence to the European Parliament (EP). The members of the EP are elected from the member states - the number of members from the individual countries are given by similar political decisions. We believe that in the European Parliament the views are stratified according to the

\footnotetext{
${ }^{1}$ For a strategic analysis of the more recent extensions see Kóczy (2010).
} 
political views of the members and national politics play a lesser role and so our focus remained on the Council of Ministers. The recently proposed Cambridge Compromise (Grimmett, 2011) studies the fair apportionment of EP seats; Barthelemy and Martin (2011) look at the apportionment in the US presidential elections. Similarly to us, these papers abstract away from the political games and evaluate the power balance mathematically. On the other hand, while the Cambridge Compromise formulates specific recommendations our aim is merely to evaluate some of the changes in the legislative process and highlight some, perhaps unintended effects.

The paper is then structured as follows. First we explain the voting rules in the Council of Ministers before and after the Lisbon Treaty. Here we also mention the Jagellonian Compromise an alternative proposal, a scientific approach to design a fair rule that was widely supported by the academic community, but got little attention in the negotiations. We explain the voting power approach, describe the data and the software we use for the calculations and present the results together with some comments.

\section{The voting reform}

The Council of Ministers is the European Union's main decision making body. The Council consists of a single representative of each member state, but when the votes are cast their weight might differ. This is different from the weighted voting in, for instance, a national assembly, where the weights are naturally provided by the number of representatives. The qualified majority voting used in the Council allows for flexible, some might say: arbitrary rules on what are the winning coalitions. In the following we explain the rules applied before and after the Reform Treaty.

\subsection{Treaty of Nice}

Under the Treaty of Nice a coalition of countries must satisfy all of the following 3 conditions in order to be able to pass a decision:

- The coalition must consist of the majority ${ }^{2}$ of countries (where each country has a single "vote").

- The coalition must cast at least $74 \%$ of the votes.

\footnotetext{
${ }^{2}$ The required majority is $50 \%$ in most cases, with the exception when the Council is not acting on a proposal of the Commission: such cases require a two-third majority.
} 
- The coalition must have at least $62 \%$ of the population.

Choosing the weights for the individual countries is not an easy task, the Treaty of Nice was preceded by long negotiations. Leech (2002) gives a detailed investigation of different extension scenarios including a discussion on the significance of quotas, that is, the required majority. Felsenthal and Machover (2001) argue that the quotas were simply set too high, paralysing EU decision making.

\subsection{Treaty of Lisbon}

The Treaty of Lisbon abolished the artificial and much debated weighted voting system and replaced it with one, based on population data. It has also increased the role of the European Parliament in the decision making process. Since voting in the EP is based on individual representatives that form voting blocks not on a national, but on political/ideological base, here we focus on voting in the Council of Ministers.

Under the new treaty the decision must be supported by a coalition of countries that satisfies at least one of the following conditions:

- The majority of countries (55\% or $72 \%$ in special cases) representing at least $65 \%$ of the population, or

- No more than 3 countries are against the proposal.

Without the second condition any 3 of the 4 largest countries could, by themselves block a proposal. While such divisions between the large and the small members of the EU do not really occur, this condition was included as a safeguard against a possible alliance of the largest members.

The Treaty specifies that until 31 October 2014 the Nice rules will be used. After this date the new rules should be used, but "Between 1 November 2014 and 31 March 2017, when an act is to be adopted by qualified majority, a member of the Council may request that it be adopted in accordance with the qualified majority..." 3 , in other words, according to the Nice rules. While secondary sources (e.g. Mahony, 2007) cite this part of the treaty in a more neutral tone: "the original sysyem [sic!] under Nice can still be used to take decisions if a member state thinks it necessary" the possibility only applies

\footnotetext{
${ }^{3}$ Treaty of Lisbon, Protocol on Transitional Provisions, Provisions concerning the qualified majority, $\S 3.2$.
} 
to the adoption of an act and not to the rejection. Should it also apply to the latter, we would be back to the Nice rules as in cases where there is a difference there is always a voter on one of the sides who finds the old rules more favourable.

\begin{tabular}{lccccc} 
& & number & population & votes \\
\hline Nice & -31 October 2014 & $50 \%$ & 14 & $62 \%$ & $74 \%$ \\
\hline \multirow{3}{*}{ Transition } & 31 October 2014 & $50 \%$ & 14 & $62 \%$ & $74 \%$ \\
& - & $55 \%$ & 15 & $65 \%$ & \\
& 31 March 2017 & $\mathrm{n}-3$ & 24 & & \\
\hline \multirow{2}{*}{ Lisbon } & \multirow{2}{*}{31 March 2017- } & $55 \%$ & 15 & $65 \%$ & \\
& & $\mathrm{n}-3$ & 24 & & \\
\hline
\end{tabular}

Table 1: A comparison of voting rules

In sum, during the transition period an act can be approved by either of the two systems.

\subsection{The "Jagiellonian Compromise"}

Penrose (1946) has shown that the one man-one vote idea of equity can best be achieved in an international decision making body by giving each voter a weight proportional to the square root of its population. This idea was advocated in the case of the EU Council of Ministers by Słomczyński and Życzkowski $(2004,2006)$ who proposed a quota of $62 \%$ as giving the most equitable representation.

Słomczyński and Życzkowski (2004) argued that the proposal does not ex ante favour any of the member states, its principles are clear and mathematically sound. The square-root model would give a scientific approach that at the same time would provide effectiveness of $16.6 \%$, that, as we will see well exceeds the decisiveness of any of the previous models. In practice this means that 1 out of 6 coalitions can make a decision. The proposal was put forward as a "compromise," as it gives a larger influence for the largest, smaller influence for medium sized countries, but not to the extent of the Lisbon rules. On the other hand for many of the member states the square-root model is actually the best and only Italy and Spain and, when we look at the Banzhaf indices, some of the smallest countries would suffer under that rule. It is therefore somewhat surprising that while the majority of countries should prefer the Jagellonian proposal, it was not seriously considered. 


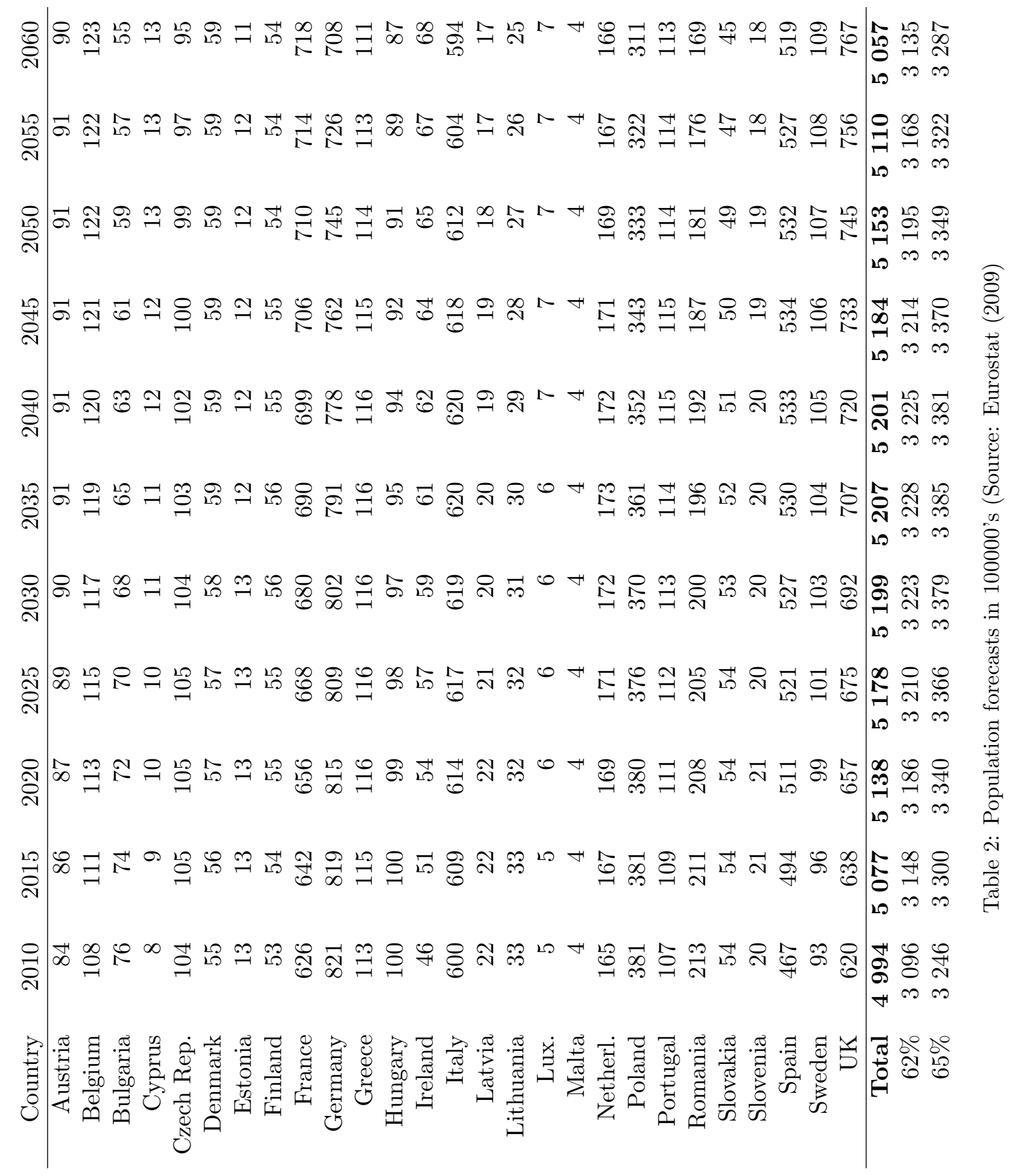




\begin{tabular}{rrrrrrr} 
& \multicolumn{2}{c}{ Shapley-Shubik index } & \multicolumn{2}{c}{ Banzhaf index } \\
& Lisbon & Penrose & Nice & Lisbon & Penrose & Nice \\
\hline Austria & $2,08 \%$ & $3,06 \%$ & $2,80 \%$ & $2,58 \%$ & $3,16 \%$ & $3,09 \%$ \\
Belgium & $2,56 \%$ & $3,56 \%$ & $3,39 \%$ & $2,92 \%$ & $3,63 \%$ & $3,68 \%$ \\
Bulgaria & $1,57 \%$ & $2,40 \%$ & $2,80 \%$ & $2,21 \%$ & $2,52 \%$ & $3,09 \%$ \\
Cyprus & $0,91 \%$ & $1,24 \%$ & $1,09 \%$ & $1,76 \%$ & $1,38 \%$ & $1,25 \%$ \\
Czech Republic & $2,17 \%$ & $3,13 \%$ & $3,39 \%$ & $2,63 \%$ & $3,22 \%$ & $3,68 \%$ \\
Denmark & $1,60 \%$ & $2,49 \%$ & $1,95 \%$ & $2,25 \%$ & $2,60 \%$ & $2,18 \%$ \\
Estonia & $0,89 \%$ & $1,15 \%$ & $1,09 \%$ & $1,74 \%$ & $1,29 \%$ & $1,25 \%$ \\
Finland & $1,52 \%$ & $2,38 \%$ & $1,95 \%$ & $2,20 \%$ & $2,50 \%$ & $2,18 \%$ \\
France & $12,47 \%$ & $8,89 \%$ & $8,75 \%$ & $9,91 \%$ & $8,41 \%$ & $7,78 \%$ \\
\hline Germany & $12,72 \%$ & $8,82 \%$ & $8,75 \%$ & $9,79 \%$ & $8,35 \%$ & $7,78 \%$ \\
Greece & $2,42 \%$ & $3,38 \%$ & $3,39 \%$ & $2,80 \%$ & $3,46 \%$ & $3,68 \%$ \\
Hungary & $2,05 \%$ & $3,00 \%$ & $3,39 \%$ & $2,55 \%$ & $3,10 \%$ & $3,68 \%$ \\
Ireland & $1,72 \%$ & $2,65 \%$ & $1,95 \%$ & $2,35 \%$ & $2,77 \%$ & $2,18 \%$ \\
Italy & $10,20 \%$ & $8,02 \%$ & $8,70 \%$ & $8,54 \%$ & $7,66 \%$ & $7,78 \%$ \\
Latvia & $0,97 \%$ & $1,39 \%$ & $1,09 \%$ & $1,81 \%$ & $1,52 \%$ & $1,25 \%$ \\
Lithuania & $1,10 \%$ & $1,67 \%$ & $1,95 \%$ & $1,89 \%$ & $1,81 \%$ & $2,18 \%$ \\
Luxembourg & $0,82 \%$ & $0,96 \%$ & $1,09 \%$ & $1,70 \%$ & $1,10 \%$ & $1,25 \%$ \\
Malta & $0,78 \%$ & $0,75 \%$ & $0,81 \%$ & $1,67 \%$ & $0,89 \%$ & $0,94 \%$ \\
\hline Spain & $8,61 \%$ & $7,46 \%$ & $8,05 \%$ & $7,68 \%$ & $7,18 \%$ & $7,42 \%$ \\
Netherlands & $3,28 \%$ & $4,13 \%$ & $3,67 \%$ & $3,36 \%$ & $4,16 \%$ & $3,97 \%$ \\
Poland & $5,53 \%$ & $5,69 \%$ & $7,98 \%$ & $4,28 \%$ & $5,60 \%$ & $7,42 \%$ \\
Portugal & $2,44 \%$ & $3,41 \%$ & $3,39 \%$ & $2,82 \%$ & $3,49 \%$ & $3,68 \%$ \\
Romania & $3,43 \%$ & $4,17 \%$ & $3,98 \%$ & $3,39 \%$ & $4,20 \%$ & $4,26 \%$ \\
Slovakia & $1,42 \%$ & $2,19 \%$ & $1,95 \%$ & $2,10 \%$ & $2,32 \%$ & $2,18 \%$ \\
\hline Kingdom & $13,40 \%$ & $9,23 \%$ & $8,75 \%$ & $10,48 \%$ & $8,69 \%$ & $7,78 \%$
\end{tabular}

Table 3: Projected power in 2060: Lisbon rules in comparison with the Penrose square-rule and the Nice status quo. 


\section{Methods}

\subsection{Power index approach}

When we study power we really study the countries' ability to alter decisions. We focus on a priori measures of voting power, that is, voting power before the issues to be voted on or the voters' positions on these issues are known. This simplification overlooks the aligned preferences of some member states (Kaniovski and Leech, 2009, provides a more general model), but considering that the actual votes cast are not made public only speculation is possible.

Depending on whether the countries's objective is to push for a particular policy change (the I-power, Felsenthal and Machover, 1998, 2004) or simply to be able to make decisions (P-power) we must use a different power measure. Since this is not very clear, we study both: we use the Banzhaf-index (Banzhaf, 1965; Coleman, 1971) to measure I-power and the Shapley-Shubik index (Shapley and Shubik, 1954) for the P-power. Straffin (1977) introduced a probabilistic approach and has shown that the two methods differ in whether the voters' probabilities of supporting a motion are independent or the same. Straffin, Jr. (1988) gives an extensive comparison of the two approaches even providing examples (with voting in two chambers) where even the order of players changes when ranked according to power. Here the differences are not so extreme, the Shapley-Shubik index sees large players comparatively more powerful.

\subsection{Data}

Already in the Nice rules populations are mentioned as a condition for a coalition to be winning, but in the Lisbon rules populations take the leading role to determine a country's influence in voting. In our estimates of voting power we use the population forecasts of Eurostat, the statistical institute of the European Union. "Eurostat's population projections is one of several possible population change scenarios based on assumptions for fertility, mortality and migration. The method used for population projections is the "cohort-component" method" (Eurostat, 2009) that divides the population into age-sex cohorts and accounts for the fertility, mortality, and migration behaviour of each of these cohorts. (George et al., 2004)

The forecasts are available for every 5 years between 2010 and 2060 and are shown in Table 2. 


\subsection{Software}

The calculations are made using Indices of Power (IOP) 2.0 (Bräuninger and König, 2005). Indices of Power is a well-established, trusted program that calculates various power indices and measures including the ShapleyShubik index and the normalised Banzhaf measure for a broad class of weighted voting situations with several criteria and at most 10 chambers and 50 actors in each. As such it is a natural if not the only candidate to calculate power indices in complex voting situations.

Originally written to handle weighted voting where the main roles have been played by the voting weights, normally small integers so Indices of Power cannot handle large weights such as populations. As a compromise population data are entered in 100000's that may have a minor effect on the indices found.

\begin{tabular}{rrrrrrrrr} 
& \multicolumn{4}{c}{ Shapley-Shubik index } & \multicolumn{3}{c}{ Normalised Banzhaf value } \\
& 2010 & 2015 & 2020 & 2060 & 2010 & 2015 & 2020 & 2060 \\
\hline Bulgaria & 2,81 & 1,81 & 1,79 & 1,57 & 3,09 & 2,40 & 2,34 & 2,21 \\
France & 8,71 & 11,17 & 11,20 & 12,47 & 7,78 & 8,98 & 9,17 & 9,91 \\
Germany & 8,76 & 15,04 & 15,12 & 12,72 & 7,78 & 11,31 & 10,98 & 9,79 \\
Hungary & 3,40 & 2,21 & 2,20 & 2,05 & 3,68 & 2,71 & 2,67 & 2,55 \\
Lithuania & 1,95 & 1,19 & 1,18 & 1,10 & 2,18 & 1,92 & 1,90 & 1,89 \\
Malta & 0,81 & 0,75 & 0,75 & 0,78 & 0,94 & 1,57 & 1,57 & 1,67 \\
Poland & 7,99 & 6,52 & 6,53 & 5,53 & 7,42 & 5,35 & 5,05 & 4,28 \\
U. K. & 8,71 & 11,09 & 11,11 & 13,40 & 7,78 & 8,93 & 9,26 & 10,48 \\
\hline decisiveness & & & & & 2,03 & 12,80 & 12,70 & 12,71
\end{tabular}

Table 4: Power indices for selected countries in selected years

\section{Results}

We are interested in three effects. Firstly, whether and to what extent are the new voting rules able to improve the EU's decisiveness. As Hosli (2008) observed and discussed in detail, the days when the EU could make decisions by unanimity are over. Second, we want to see a better equity among European citizens. Finally any change will have winners and losers. We are also interested in the "dynamic equity" the possible loss of acquired rights. 
Note that our results have been obtained with the usual limitations inherent to a priori measures of voting power and focus solely on the voting power in the Council of Ministers. One must note that the decisions are often made with unanimity and there are clearly countries that are agenda setters that may lead to a higher share of the power. We have knowingly ignored possible new entries as we want to study the effect of the new voting rules rather than the effects of the entry.

\subsection{Improved decisiveness}

The results for the calculations are summarised in Table 4 for selected years and selected countries - for the complete dataset see Tables A.6 and A.7. Most importantly we can see that the probability of decisiveness has increased dramatically. While in the Nice system about 1 out of 50 coalitions of countries could approve a decision, under the new system this increases to more than 1 out of 8. ${ }^{4}$ Naturally, the best period is between 2014 and 2017, when either of the rules can be applied to approve a decision. The gain, however, is rather small. There are in total $2^{27}-1$ or over 134 million possible coalitions among the 27 member states, of which only about 2500 are decisive under the old, but not under the new rules. This seems like a fair price to abandon a complicated system.

\subsection{On representation}

The increased decisiveness on the other hand means that there are many (millions) of additional coalitions that are now winning. Not all members benefit from these changes equally. One of the criticisms of the old system was the enormous differences between the influence of an individual citizen depending on his citizenship. While this is hardly avoidable in such a complex situation, the severity of the problem is striking. Citizens of Luxembourg or Malta have about 20 times as much influence as citizens of Germany. Interestingly the two former members are also the largest recipients of EU funds on a per capita basis (European Parliament, 2010). Has this improved?

Before we can answer this question, we must decide on the benchmark: what would be the fair allocation of power? Penrose (1946) has in his already mentioned result established the Penrose square-root rule, stating that the

\footnotetext{
${ }^{4}$ For a reference point note that in a simple majority setting a coalition or its complementer has the majority of the votes and so the ratio is 1 to 2 .
} 
voting weights should be proportional to the square root of the populations. We have presented the power in this benchmark scenario as well as the preand post-reform powers in Table 3. It is apparent that the new power distribution is further from the ideal. Taking the (unweighted quadratic) average difference as a measure of distance, the Lisbon rules are 2 (Banzhaf index) to 7 (Shapley-Subik index) times less equal.
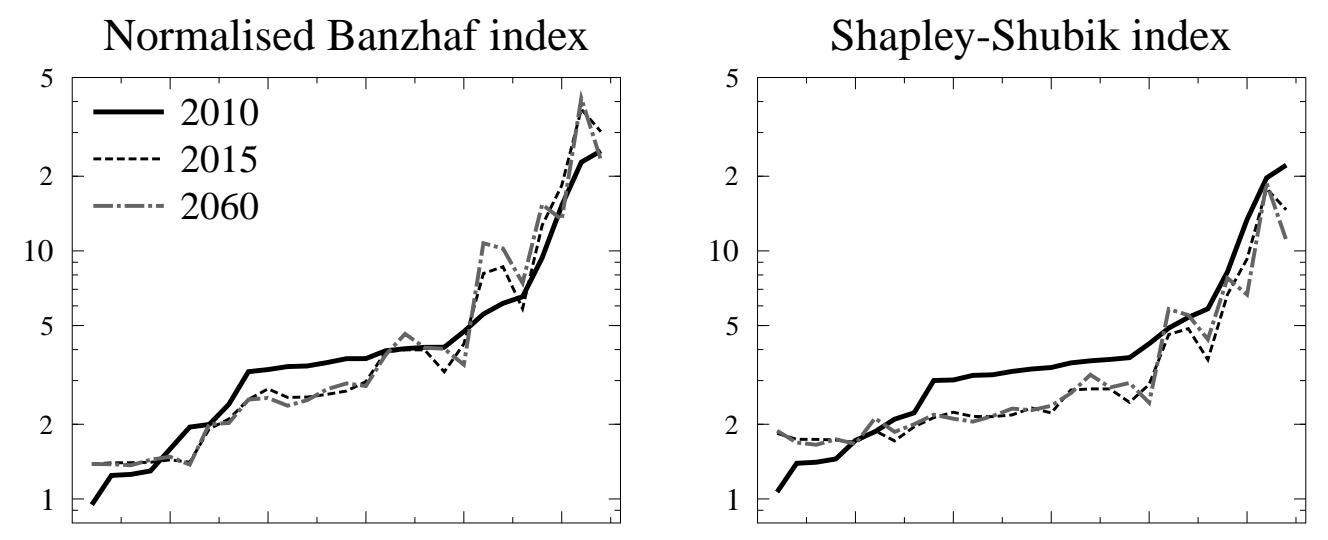

Figure 1: Representation before and after the reform: $10^{-9}$ times the probability that a member state is decisive on behalf of a particular citizen (note the logarithmic scale). Countries in decreasing order of 2010 representativeness from Germany to Luxembourg.

One could say that the Penrose square rule is politically unacceptable and so it is also interesting to see a comparison with another benchmark, where we want to have power to be proportional to the populations. So let us see how the reform fares with respect to this benchmark that the decision makers may have used, when representativeness is measured by the probability that a member states makes a decision on behalf of a particular citizen. Here the conclusions are somewhat different when studying the Shapley-Shubik (Table A.6) or normalised Banzhaf indices (Tables A.7). Looking at the first in Figure 1, we find that the overall changes are very favourable: the representation of Germany dramatically improves, and although Malta's does not decrease much, Maltese citizens will have only about 10 times as much influence as the citizens of a larger country. Medium sized countries are clear losers compared to the status quo, but as a result the top 20 countries are very similarly represented with less than a factor of 1.8 in representativeness. In the case of the Banzhaf index, yet again, Germany's representation improves 
much, overtaking France, Poland and the UK, too, but smaller countries become better represented, so that by 2060 Maltese citizens have over 30 times the influence of their British peers. It could well be that we talk about some outliers. Unfortunately this is not the case: the (unweighted quadratic) average distance from equal representation more than doubles for the Banzhaf index.

\begin{tabular}{rrrrrrrr} 
& \multicolumn{3}{c}{ Shapley-Shubik } & \multicolumn{3}{c}{ Banzhaf } \\
& reform & pop. & total & & reform & pop. & total \\
\hline U. K. & $27 \%$ & $21 \%$ & $54 \%$ & Malta & $67 \%$ & $6 \%$ & $77 \%$ \\
Germany & $72 \%$ & $-15 \%$ & $45 \%$ & Slovenia & $42 \%$ & $3 \%$ & $45 \%$ \\
France & $28 \%$ & $12 \%$ & $43 \%$ & Latvia & $43 \%$ & $1 \%$ & $45 \%$ \\
Italy & $21 \%$ & $-3 \%$ & $17 \%$ & Cyprus & $30 \%$ & $8 \%$ & $41 \%$ \\
$\ldots$ & $\ldots$ & $\ldots$ & $\ldots$ & Estonia & $34 \%$ & $4 \%$ & $39 \%$ \\
Luxembourg & $-30 \%$ & $7 \%$ & $-25 \%$ & Luxembourg & $26 \%$ & $8 \%$ & $36 \%$ \\
Austria & $-29 \%$ & $4 \%$ & $-26 \%$ & U. K. & $15 \%$ & $17 \%$ & $35 \%$ \\
Portugal & $-23 \%$ & $-6 \%$ & $-27 \%$ & France & $15 \%$ & $10 \%$ & $27 \%$ \\
Slovakia & $-31 \%$ & $4 \%$ & $-28 \%$ & Germany & $45 \%$ & $-13 \%$ & $26 \%$ \\
Greece & $-28 \%$ & $-1 \%$ & $-29 \%$ & $\ldots$ & $\ldots$ & $\ldots$ & $\ldots$ \\
Poland & $-18 \%$ & $-15 \%$ & $-31 \%$ & Greece & $-22 \%$ & $-3 \%$ & $-24 \%$ \\
Czech Rep. & $-33 \%$ & $-5 \%$ & $-36 \%$ & Bulgaria & $-22 \%$ & $-8 \%$ & $-29 \%$ \\
Hungary & $-35 \%$ & $-7 \%$ & $-40 \%$ & Czech Rep. & $-25 \%$ & $-5 \%$ & $-29 \%$ \\
Lithuania & $-39 \%$ & $-8 \%$ & $-44 \%$ & Hungary & $-26 \%$ & $-6 \%$ & $-31 \%$ \\
Bulgaria & $-36 \%$ & $-13 \%$ & $-44 \%$ & Poland & $-28 \%$ & $-20 \%$ & $-42 \%$
\end{tabular}

Table 5: The most drastic relative changes in the power indices. (The effect of the population change refers to the period until 2060.)

This is hardly surprising. Firstly note that the power $\kappa_{i}$ of a player can be expressed as a weighted average of her power or contribution $\mu_{i}$ in the winning coalitions (Kóczy, 2008).

$$
\kappa_{i}=\sum_{C \in 2^{N} \backslash \varnothing} a^{C} \mu_{i}^{C}
$$

where $\sum_{C \in 2^{N} \backslash \varnothing} a^{C}=1$.

Within a coalition the contributions $\mu_{i}$ are treated equally by the two measures, what differs are the weights $a^{C}$. For the Shapley-Shubik index

$$
a_{\phi}^{C}=\frac{(|C|-1) ! k^{C}(n-|C|) !}{n !} .
$$


while for the Banzhaf index

$$
a_{\beta}^{C}=\frac{k^{C}}{\sum_{C \in \mathcal{W}} k^{C}} .
$$

where $k^{C}$ is the number of critical players in coalition $C$.

The difference in the indices is most pronounced for large coalitions. When such a large coalition is a minimal winning coalition, it contains many small members, each of which are critical. Large coalitions get a high weight for Shapley-Shubik, and a coalition with many critical players gets a high weight for Banzhaf. On the other hand, a large surplus coalition has a few critical large voters: the weight of the coalition is still large for ShapleyShubik, but due to the fewer critical players, (much) smaller for Banzhaf.

Under the Nice rules the number and population of voters rarely mattered and voting was decided based on the voting weights. Voting weights represented a compromise between size and number: in the new system this is not necessary. When a coalition lacks population, a large voter can help a lot. Similarly, when the population is there, but the coalition is not sufficiently inclusive, even the smallest member states are useful, in fact equally useful as the bigger ones. Medium sized voters turn out to be not much more needed than small voters, while a lot less useful as the big ones. On the other hand the weighting method of the two measures explains why are large players who benefit from the reform most according to the Shapley-Shubik index, while according to the Banzhaf measure small member states gain even more.

\subsection{Winners and losers}

As long as we are interested in shares of voting power, any change will benefit some member countries and harm others. Who the winners and losers are depends on the details of the change (Baldwin and Widgrén, 2004). In the following we look at the effects of the voting reform.

The changes we have so far discussed are due to the change in the voting system, but also due to population changes. Bulgaria, Latvia and Lithuania lose around a quarter of their population, the UK gains that much, while Cyprus, Luxembourg and Ireland are predicted to gain more than $50 \%$ of theirs. The effect of the new system, observable at the 2015 values is drastic for the largest countries, Germany gaining nearly $80 \%$, while some middlesized countries: Bulgaria, Hungary, Lithuania lose the most 35-40\% of their power for the Shapley-Shubik index. If we look at the normalised Banzhaf, 
the largest and the smallest countries gain, the biggest losses are recorded for Hungary, Poland, the Czech Republic. Overall the same countries, together with Bulgaria lose the most power. While Poland and the Czech Republic were among the last ones, interestingly Hungary was the first one to ratify the treaty, it took her no more than 9 days and less than an hour of discussion.

Arguably these changes are not due to the reform, but to unfavourable demographic changes. This may be true, but before the reform the populations rarely mattered and the population forecasts were known at the time of signing the treaty.

Finally we make two observations. Firstly, the effects are sometimes just the opposite for Shapley-Shubik and Banzhaf indices. Luxembourg is an example that loses according to Shapley-Shubik, but wins according to Banzhaf. Estonia is a country with a slowly but steadily decreasing population, yet its Banzhaf power has the opposite trend.

Second, it is interesting to note, how certain groups of countries benefit more than others. The biggest losers of the reform are all recipients from the budget. Based on the Shapley-Shubik indices the share of the biggest per capita recipients goes down from $40 \%$ to $28 \%$, the major contributors gain power from $29 \%$ to $35 \%$. Looking at other groups, we find that the 4 largest countries presently having about a third of the influence increase their share to nearly half. Central and East European Countries (the Visegrád countries together with Romania and Bulgaria) presently decide about 1 in 4 decisions. By 2060 this share goes down to 1 in 6 looking at the current trends.

\section{Conclusions}

One of the objectives of the Treaty of Lisbon was to reform the slow, increasingly impotent decision making of the European Union. This task was completed with success making it about 6 times easier to reach a decision. The changes were also made with the intention to make the decision making more fair, allowing all European citizens to have the same influence on the decision making. Here the success was more moderate halving the past inequalities even if we look at the Shapley-Shubik index, while the normalised Banzhaf value reports a worsening of the situation.

The changes do not affect all countries equally. The large countries dominate European politics - the rules of the Lisbon Treaty have been mediated by Germany. The base+prop method of the Cambridge Compromise (Grimmett, 2011) favours the largest and the smallest member states. Here, too, 
the large countries are clear beneficiaries of the changes: the mechanisms to limit their influence have been mostly removed. The countries that suffer most from the changes are surprisingly not the smallest ones, but the countries in Central and Eastern Europe, where a combination of the reform together with unfavourable demographic changes mean that in 50 years they will have much less influence than what they have today.

\section{Appendix A. Tables}

\begin{tabular}{lrrrrrrrrrr} 
& 2010 & 2015 & 2020 & 2030 & 2035 & 2040 & 2045 & 2050 & 2055 & 2060 \\
\hline Austria & 2,81 & 1,99 & 1,98 & 1,98 & 2,02 & 2,04 & 2,05 & 2,06 & 2,07 & 2,08 \\
Belgium & 3,40 & 2,38 & 2,37 & 2,38 & 2,44 & 2,47 & 2,49 & 2,52 & 2,55 & 2,56 \\
Bulgaria & 2,81 & 1,81 & 1,79 & 1,75 & 1,70 & 1,66 & 1,63 & 1,61 & 1,59 & 1,57 \\
Cyprus & 1,10 & 0,82 & 0,82 & 0,83 & 0,85 & 0,86 & 0,88 & 0,88 & 0,90 & 0,91 \\
Czech Republic & 3,40 & 2,29 & 2,28 & 2,26 & 2,24 & 2,22 & 2,22 & 2,20 & 2,19 & 2,17 \\
Denmark & 1,95 & 1,54 & 1,53 & 1,53 & 1,55 & 1,57 & 1,57 & 1,58 & 1,59 & 1,60 \\
Estonia & 1,10 & 0,88 & 0,88 & 0,88 & 0,88 & 0,87 & 0,88 & 0,88 & 0,89 & 0,89 \\
Finland & 1,95 & 1,51 & 1,50 & 1,50 & 1,52 & 1,52 & 1,51 & 1,52 & 1,51 & 1,52 \\
France & 8,71 & 11,17 & 11,20 & 11,31 & 11,60 & 11,77 & 11,95 & 12,11 & 12,28 & 12,47 \\
Germany & 8,76 & 15,04 & 15,12 & 14,79 & 14,23 & 13,95 & 13,64 & 13,32 & 13,03 & 12,72 \\
Greece & 3,40 & 2,44 & 2,43 & 2,43 & 2,42 & 2,42 & 2,43 & 2,43 & 2,43 & 2,42 \\
Hungary & 3,40 & 2,21 & 2,20 & 2,17 & 2,13 & 2,10 & 2,09 & 2,07 & 2,07 & 2,05 \\
Ireland & 1,95 & 1,46 & 1,45 & 1,49 & 1,56 & 1,60 & 1,62 & 1,65 & 1,68 & 1,72 \\
Italy & 8,70 & 10,53 & 10,54 & 10,49 & 10,40 & 10,38 & 10,36 & 10,33 & 10,28 & 10,20 \\
Latvia & 1,10 & 1,01 & 1,01 & 1,01 & 0,99 & 0,99 & 0,98 & 0,98 & 0,98 & 0,97 \\
Lithuania & 1,95 & 1,19 & 1,18 & 1,16 & 1,15 & 1,13 & 1,12 & 1,12 & 1,11 & 1,10 \\
Luxembourg & 1,10 & 0,77 & 0,76 & 0,77 & 0,78 & 0,78 & 0,80 & 0,81 & 0,81 & 0,82 \\
Malta & 0,81 & 0,75 & 0,75 & 0,74 & 0,75 & 0,75 & 0,76 & 0,76 & 0,77 & 0,78 \\
Netherlands & 3,67 & 3,26 & 3,27 & 3,28 & 3,32 & 3,33 & 3,32 & 3,32 & 3,30 & 3,28 \\
Poland & 7,99 & 6,52 & 6,53 & 6,45 & 6,21 & 6,06 & 5,92 & 5,80 & 5,67 & 5,53 \\
Portugal & 3,40 & 2,35 & 2,34 & 2,35 & 2,38 & 2,39 & 2,42 & 2,43 & 2,43 & 2,44 \\
Romania & 3,98 & 3,97 & 4,01 & 3,93 & 3,77 & 3,71 & 3,65 & 3,58 & 3,50 & 3,43 \\
Slovakia & 1,95 & 1,51 & 1,50 & 1,49 & 1,47 & 1,46 & 1,45 & 1,44 & 1,44 & 1,42 \\
Slovenia & 1,10 & 1,00 & 0,99 & 0,99 & 0,99 & 0,99 & 0,99 & 0,98 & 0,99 & 0,98 \\
Spain & 8,04 & 8,35 & 8,34 & 8,51 & 8,61 & 8,62 & 8,64 & 8,65 & 8,64 & 8,61 \\
Sweden & 2,81 & 2,14 & 2,13 & 2,17 & 2,22 & 2,24 & 2,26 & 2,29 & 2,31 & 2,34 \\
UK & 8,71 & 11,09 & 11,11 & 11,33 & 11,84 & 12,12 & 12,38 & 12,68 & 13,03 & 13,40
\end{tabular}

Table A.6: Shapley-Shubik indices of the member countries (in \%) 


\begin{tabular}{lrrrrrrrrrr} 
& 2010 & 2015 & 2020 & 2030 & 2035 & 2040 & 2045 & 2050 & 2055 & 2060 \\
\hline Austria & 3,09 & 2,55 & 2,56 & 2,57 & 2,58 & 2,57 & 2,58 & 2,58 & 2,58 & 2,58 \\
Belgium & 3,68 & 2,84 & 2,86 & 2,87 & 2,89 & 2,89 & 2,90 & 2,90 & 2,90 & 2,92 \\
Bulgaria & 3,09 & 2,40 & 2,34 & 2,32 & 2,29 & 2,27 & 2,25 & 2,23 & 2,22 & 2,21 \\
Cyprus & 1,25 & 1,63 & 1,65 & 1,67 & 1,68 & 1,70 & 1,72 & 1,74 & 1,75 & 1,76 \\
Czech Republic & 3,68 & 2,77 & 2,75 & 2,73 & 2,71 & 2,69 & 2,67 & 2,66 & 2,64 & 2,63 \\
Denmark & 2,18 & 2,19 & 2,19 & 2,21 & 2,22 & 2,22 & 2,23 & 2,23 & 2,24 & 2,25 \\
Estonia & 1,25 & 1,68 & 1,68 & 1,69 & 1,69 & 1,70 & 1,72 & 1,73 & 1,74 & 1,74 \\
Finland & 2,18 & 2,17 & 2,17 & 2,18 & 2,18 & 2,18 & 2,18 & 2,18 & 2,19 & 2,20 \\
France & 7,78 & 8,98 & 9,17 & 9,28 & 9,39 & 9,51 & 9,61 & 9,71 & 9,81 & 9,91 \\
Germany & 7,78 & 11,31 & 10,98 & 10,82 & 10,65 & 10,47 & 10,29 & 10,13 & 9,95 & 9,79 \\
Greece & 3,68 & 2,89 & 2,87 & 2,86 & 2,85 & 2,85 & 2,83 & 2,82 & 2,81 & 2,80 \\
Hungary & 3,68 & 2,71 & 2,67 & 2,65 & 2,62 & 2,61 & 2,59 & 2,58 & 2,56 & 2,55 \\
Ireland & 2,18 & 2,13 & 2,19 & 2,22 & 2,24 & 2,26 & 2,28 & 2,30 & 2,33 & 2,35 \\
Italy & 7,78 & 8,56 & 8,55 & 8,55 & 8,58 & 8,60 & 8,62 & 8,61 & 8,59 & 8,54 \\
Latvia & 1,25 & 1,78 & 1,77 & 1,77 & 1,78 & 1,78 & 1,79 & 1,79 & 1,79 & 1,81 \\
Lithuania & 2,18 & 1,92 & 1,90 & 1,90 & 1,89 & 1,89 & 1,89 & 1,89 & 1,89 & 1,89 \\
Luxembourg & 1,25 & 1,58 & 1,60 & 1,61 & 1,62 & 1,65 & 1,66 & 1,67 & 1,69 & 1,70 \\
Malta & 0,94 & 1,57 & 1,57 & 1,59 & 1,60 & 1,61 & 1,63 & 1,64 & 1,65 & 1,67 \\
Netherlands & 3,97 & 3,51 & 3,51 & 3,50 & 3,48 & 3,45 & 3,43 & 3,39 & 3,37 & 3,36 \\
Poland & 7,42 & 5,35 & 5,05 & 4,91 & 4,76 & 4,61 & 4,49 & 4,39 & 4,32 & 4,28 \\
Portugal & 3,68 & 2,82 & 2,83 & 2,83 & 2,83 & 2,84 & 2,83 & 2,82 & 2,82 & 2,82 \\
Romania & 4,26 & 4,04 & 3,91 & 3,82 & 3,74 & 3,67 & 3,60 & 3,52 & 3,46 & 3,39 \\
Slovakia & 2,18 & 2,17 & 2,16 & 2,15 & 2,14 & 2,13 & 2,13 & 2,13 & 2,11 & 2,10 \\
Slovenia & 1,25 & 1,77 & 1,76 & 1,77 & 1,78 & 1,79 & 1,79 & 1,80 & 1,80 & 1,82 \\
Spain & 7,42 & 7,10 & 7,36 & 7,42 & 7,49 & 7,56 & 7,63 & 7,68 & 7,71 & 7,68 \\
Sweden & 3,09 & 2,66 & 2,70 & 2,72 & 2,72 & 2,73 & 2,74 & 2,75 & 2,76 & 2,78 \\
UK & 7,78 & 8,93 & 9,26 & 9,43 & 9,61 & 9,76 & 9,94 & 10,13 & 10,31 & 10,48 \\
\hline decisiveness & 2,03 & 12,80 & 12,70 & 12,71 & 12,69 & 12,69 & 12,69 & 12,67 & 12,67 & 12,71
\end{tabular}

Table A.7: The normalised Banzhaf indices and the decisiveness (in \%) 


\section{References}

Baldwin, R., Widgrén, M., 2004. Winners and Losers Under Various Dual Majority Rules for the EU Council of Ministers. CEPR Discussion Paper 4450. Centre for Economic Policy Research. London.

Banzhaf, III., J.F., 1965. Weighted voting doesn't work: A mathematical analysis. Rutgers Law Review 19, 317-343.

Barr, J., Passarelli, F., 2009. Who has the power in the EU? Mathematical Social Sciences 57, 339-366.

Barthelemy, F., Martin, M., 2011. A comparison between the methods of apportionment using power indices: the case of the U.S. presidential elections.

Bräuninger, T., König, T., 2005. Indices of Power IOP 2.0 [computer program]. University of Konstanz. Konstanz.

Coleman, J.S., 1971. Control of collectives and the power of a collectivity to act, in: Lieberman, B. (Ed.), Social Choice. Gordon and Breach, New York, pp. 192-225.

European Parliament, 2010. Definitive adoption of the european union's general budget for the financial year 2010. Official Journal of the European Union 53, 1 .

Eurostat, 2009. Europop2008 - convergence scenario, national level, population predictions - [tps00002].

Felsenthal, D.S., Machover, M., 1998. The Measurement of Voting Power: Theory and Practice, Problems and Paradoxes. Edward Elgar, Cheltenham.

Felsenthal, D.S., Machover, M., 2001. The treaty of Nice and qualified majority voting. Social Choice and Welfare 18, 431-464.

Felsenthal, D.S., Machover, M., 2004. A priori voting power: What is it all about? Political Studies Review 2, 1-23. 
Felsenthal, D.S., Machover, M., 2007. Analysis of QM Rule adopted by the Council of the European Union, Brussels, 23 June 2007. Eprint 2531. London School of Economics. London.

George, M.V., Smith, S.K., Swanson, D.A., Tayman, J., 2004. Population projections, in: Siegel, J., Swanson, D. (Eds.), The Methods and Materials of Demography. Elsevier, San Diego. chapter 21.

Grimmett, G.R., 2011. European Apportionment via the Cambrdige Compromise. Mathematical Social Sciences .

Hosli, M.O., 2008. Council decision rules and european union constitutional design. AUCO Czech Economic Review 2, 76-96.

Kaniovski, S., Leech, D., 2009. A behavioral power index. Public Choice $141,17-29$.

Kóczy, L.Á., 2008. Strategic power indices: Quarrelling in coalitions. Working paper 0803. Budapest Tech, Keleti Faculty of Economics. Budapest.

Kóczy, L.Á., 2010. Strategic aspects of the 1995 and 2004 EU enlargements. Group Decision and Negotiation 19, 267-277.

Leech, D., 2002. Designing the voting system for the council of the european union. Public Choice 113, 437-434.

Mahony, H., 2007. Eu leaders scrape treaty deal at 11th hour. EU Observer 23 June 2007.

Penrose, L.S., 1946. The elementary statistics of majority voting. Journal of the Royal Statistical Society 109, 53-57.

Shapley, L.S., Shubik, M., 1954. A method for evaluating the distribution of power in a committee system. American Political Science Review 48, $787-792$.

Słomczyński, W., Życzkowski, K., 2004. Voting in the European Union: The square root system of Penrose and a critical point. cond-mat 0405396v2. arXiv.org. Warsaw.

Słomczyński, W., Życzkowski, K., 2006. Penrose voting system and optimal quota. Acta Physica Polonica B 37, 3133. 
Straffin, Jr., P.D., 1977. Homogeneity, independence and power indices. Public Choice 30, 107-118.

Straffin, Jr., P.D., 1988. The Shapley-Shubik and Banzhaf power indices as probabilities, in: Roth, A.E. (Ed.), The Shapley value: essays in honor of Lloyd S. Shapley. Cambridge University Press, Cambridge. chapter 5, pp. 71-82.

Turnovec, F., 2008. National, political and institutional influence in european union decision making. AUCO Czech Economic Review 2, 154-173. 


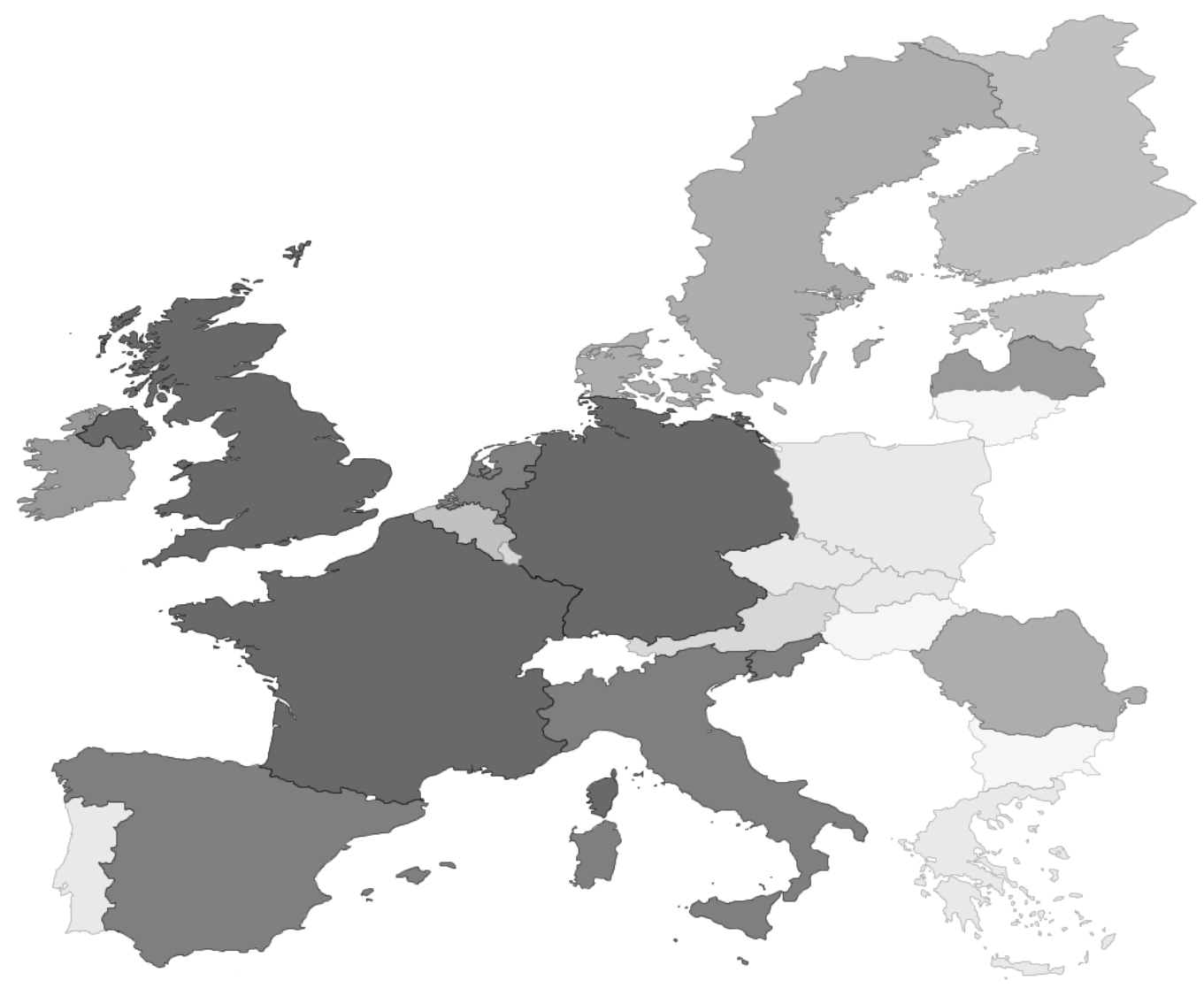

Figure 2: Cartogram showing the relative change in Shapley-Shubik indices. The area of each country is scaled by the relative change of power. Darker shades indicate a higher ratio. Note that the areas of Poland and Germany should be similar or that Bulgaria should be Latvia twice. 